\title{
Jejunal diverticulosis
}

Tetsuya Ohira ${ }^{1}$, Atsushi Iraha ${ }^{2}$, Tetsu Kinjo ${ }^{1}$, Akira Hokama ${ }^{1}$, and Jiro Fujita ${ }^{2}$

Departments of ${ }^{1}$ Endoscopy and ${ }^{2}$ Infectious, Respiratory, and Digestive Medicine, Graduate School of Medicine, University of the Ryukyus, Okinawa, Japan

Received: June 20, 2018

Revised : June 29, 2018

Accepted: June 29, 2018

\section{Correspondence to}

Akira Hokama, M.D.

Tel: +81-98-895-1144

Fax: +81-98-895-1414

E-mail: hokama-a@med.u-ryukyu.ac.jp
An 82-year-old female presented with melena. She had been on thrice-weekly conventional hemodialysis for 8 years. An esophagogastroduodenosopy and colonoscopy revealed no bleeding source. A small bowel series revealed multiple round-shaped jejunal diverticula (Fig. 1) and balloon enteroscopy confirmed large diverticula in the jejunum (Fig. 2). Diverticular bleeding was clinically suspected despite the absence of bleeding stigmata and she improved conservatively.

Symptomatic jejunal diverticular diseases including bleeding and diverticulitis are extremely rare. Recently, balloon enteroscopy has been established as a safe and effective modality to detect and treat jejunal diverticular bleeding. When a bleeding diverticulum is identified, bleeding can be managed with endoscopic clipping or injection therapy. Angiography is also useful for the diagnosis and treatment. In cases of failure of endoscopic and angiograph- ic management, surgical intervention should be considered.

Informed consent was obtained.

\section{Conflict of interest}

No potential conflict of interest relevant to this article was reported.

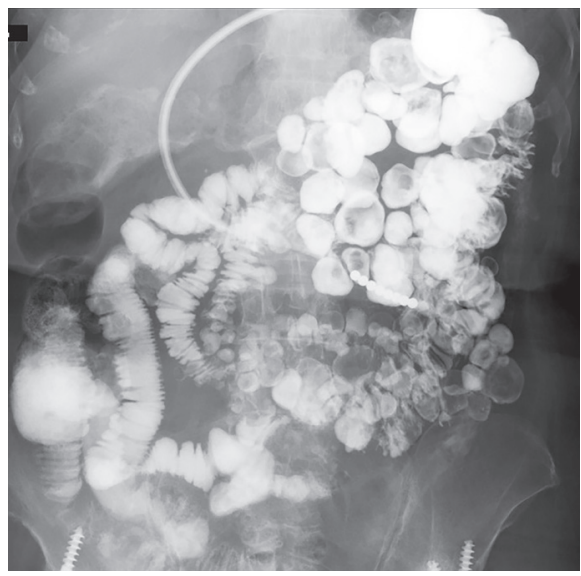

Figure 1. A small bowel series showing jejunal diverticulosis.
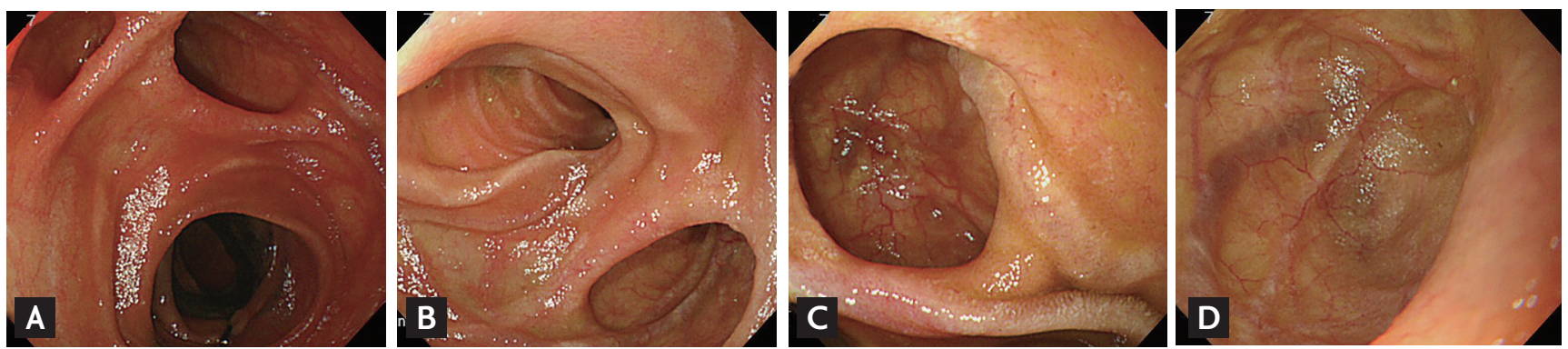

Figure 2. (A) Balloon enteroscopy reveling large diverticula in the jejunum. (B) The intestinal lumen (the left side) had Kerckring folds. (C) The vessels were shown inside the diverticulum. (D) The closer observation revealed intramucosal bold vessels on the diverticular base. 